\title{
Changing Italian nosocomial-community trends and heteroresistance in Staphylococcus aureus from bacteremia and endocarditis
}

\author{
F. Campanile • D. Bongiorno • M. Falcone • F. Vailati • M. B. Pasticci • M. Perez • \\ A. Raglio • F. Rumpianesi - C. Scuderi • F. Suter $\cdot$ M. Venditti • C. Venturelli • \\ V. Ravasio $\cdot$ M. Codeluppi $\cdot$ S. Stefani
}

Received: 23 May 2011 / Accepted: 14 July 2011 /Published online: 7 August 2011

(C) The Author(s) 2011. This article is published with open access at Springerlink.com

\begin{abstract}
Bloodstream infections due to Staphylococcus aureus (BSI) are serious infections both in hospitals and in the community, possibly leading to infective endocarditis (IE). The use of glycopeptides has been recently challenged by various forms of low-level resistance. This study evaluated the distribution of MSSA and MRSA isolates from BSI and IE in 4 Italian hospitals, their antibiotic susceptibility - focusing on the emergence of hVISA — and
\end{abstract}

F. Campanile $\cdot$ D. Bongiorno $\cdot$ M. Perez $\cdot$ C. Scuderi $\cdot$

S. Stefani $(\bowtie)$

Department of Bio-Medical Sciences, Section of Microbiology,

University of Catania,

Via Androne 81,

95124 Catania (I), Italy

e-mail: stefanis@unict.it

M. Falcone $\cdot$ M. Venditti

Department of Public Health and Infectious Disease,

Policlinico Umberto I, La Sapienza,

Rome, Italy

F. Rumpianesi $\cdot$ C. Venturelli $\cdot$ M. Codeluppi

Microbiology And Virology Laboratory,

Policlinico Hospital Of Modena,

Modena, Italy

\section{B. Pasticci}

Infectious Disease Section, Department of Experimental Medicine and Biochemical Sciences, University of Perugia,

Perugia, Italy

F. Vailati $\cdot$ A. Raglio $\cdot$ V. Ravasio

USC Microbiologia e Virologia, AO Ospedali Riuniti,

Bergamo, Italy

F. Suter

USC Malattie infettive, AO Ospedali Riuniti,

Bergamo (I), Italy genotypic relationships. Our results demonstrate that the epidemiology of MRSA is changing versus different STs possessing features between community-acquired (CA)and hospital-acquired (HA)-MRSA groups; furthermore, different MSSA isolated from BSI and IE were found, with the same backgrounds of the Italian CA-MRSA. The hVISA phenotype was very frequent (19.5\%) and occurred more frequently in isolates from IE and in both the MSSA and MRSA strains. As expected, hVISA were detected in MRSA with vancomycin minimum inhibitory concentrations (MICs) of 1-2 mg/l, frequently associated with the major SCCmec I and II nosocomial clones; this phenotype was also detected in some MSSA strains. The few cases of MR-hVISA infections evaluated in our study demonstrated that 5 out of 9 patients (55\%) receiving a glycopeptide, died. Future studies are required to validate these findings in terms of clinical impact.

\section{Introduction}

Staphylococcus aureus has become an increasing cause of nosocomial and community acquired bloodstream infections (BSI), possibly leading to infective endocarditis (IE), with a high risk of mortality despite aggressive therapy [1, 2]. Since 1990, the incidence of S.aureus bacteremia has increased because of the extensive use of indwelling intravenous catheters. Predisposing factors for $S$. aureus infections include severe underlying conditions, prolonged hospital stay, previous antibiotic treatment and nasal carriage. In this context, the emergence of methicillinresistant S.aureus (MRSA) is a major clinical challenge, particularly for the poor outcome related to such serious infections, and for the increasing therapeutic failures. In 
fact, the use of glycopeptides has been recently challenged by various forms of reduced-susceptibility (VISA and hVISA phenotypes), with consequential effects on vancomycin efficacy in MRSA bacteremia and endocarditis [3-5].

Until now, only a few studies have compared the clinical and molecular features of MSSA versus MRSA in patients with infective endocarditis or bacteremia [612]. Additional knowledge could be useful to understand and correlate the impact of specific genotypic markers with clinical outcomes.

This study was undertaken to evaluate MSSA and MRSA distribution in strains from BSI and IE isolated in four Italian hospitals, in order to evaluate their genotypic relationship, $p v l$ gene distribution, antibiotic susceptibility patterns and presence of hVISA strains.

\section{Materials and methods}

Microbial population and epidemiological correlations

The microbial population consisted of $128 \mathrm{~S}$. aureus clinical isolates, belonging to 76 patients with definite $S$. aureus IE, according to the modified Duke criteria [13], and 52 patients with definite BSI. Patients were admitted to four Italian hospitals (Modena, Bergamo, Perugia, and Rome) between 2007 and 2009. The S. aureus isolates, randomly selected (multiple isolates from the same patient and from other patients at the same time in the same ward were excluded) among all $S$. aureus isolates, were sent to our laboratory for further characterizations. Infection classification was performed as follows: nosocomial infection was defined as an IE developing in a patient hospitalized for $>48 \mathrm{~h}$ before the onset of signs and symptoms consistent with IE, and non-nosocomial health-care-associated infection was defined as an IE diagnosed within $48 \mathrm{~h}$ of admission in an outpatient with extended health-care contact. Persistent bacteremia was defined as $>3$ days of bacteremia despite receipt of an antibiotic to which the isolate was susceptible in vitro [14].

Microbiological characterization

Both groups of strains (BSI and IE) were all isolated from blood cultures. All staphylococci were re-identified at the species level by the catalase test, the $S$. aureus agglutination test (Staphylase Test; Oxoid, Basingstoke, Hampshire, UK) and biochemical tests (API-Staph system; bioMérieux, Bagno a Ripoli, FI, Italy). Methicillin resistance was evaluated by the cefoxitin disk diffusion method and correlated with the presence of the mecA gene [15-17].
Antimicrobial susceptibility was determined by the disk diffusion method, according to CLSI guidelines [15]. All isolates were tested against a panel of nine antimicrobial agents as follows: ampicillin $-1 \mu \mathrm{g}$, ciprofloxacin $-5 \mu \mathrm{g}$, chloramphenicol $-30 \mu \mathrm{g}$, gentamicin $-10 \mu \mathrm{g}$, erythromycin $-15 \mu \mathrm{g}$, clindamycin $-2 \mu \mathrm{g}$, trimethoprim-sulfamethoxazole $-25 \mu \mathrm{g}$, rifampin $-5 \mu \mathrm{g}$, and tetracycline $-30 \mu \mathrm{g}$ (Oxoid, Milan, Italy).

In vitro susceptibility testing for vancomycin (Sigma Chemical, St. Louis, MO, USA), teicoplanin, quinupristin/dalfopristin (Aventis, West Malling, UK), linezolid (Pfizer, Groton, CT, USA), tigecycline (Wyeth Pharmaceuticals, Collegeville, PA, UK) and daptomycin (Novartis, Basel, Switzerland) was further performed by the broth microdilution method to determine the minimum inhibitory concentrations (MICs), following the CLSI guidelines. The EUCAST guidelines were also used for comparison $[15,16]$. Heteroresistance to glycopeptides was screened using the macro Etest (bioMérieux), and confirmed by the reference PAP/AUC method. S. aureus Mu3 (hVISA), Mu50 (VISA), and ATCC 29213 were used as control strains, as previously described [18].

Molecular characterization of all strains was conducted by PCR of mecA and $p v l$ genes, SCCmec-typing, and MLST; PFGE was also used only to define possible relationships among the isolates. All techniques were performed as previously described [17]. MLST was performed on all MRSA strains and on a selection of MSSA isolates $(n=50)$, based on phenotypic, genotypic, and susceptibility testing differences (http://saureus.mlst.net).

\section{Clinical data on therapies for hVISA infections}

All centers were asked to provide clinical data on the treatment and outcome of patients with hVISA infections. Overall, complete data were available for 20 out of 25 patients.

\section{Results}

Strain characteristics and infections

Overall, MSSA isolates were predominant, both in BSI and IE, with only 32 out of 128 patients infected by MRSA (Table 1). In particular, 41 out of 52 patients with bacteremia were infected by MSSA (79\%), while 11 by MRSA $(21 \%)$; 55 out of 76 patients with definite $S$. aureus IE, were infected by MSSA (72\%), while 21 by MRSA (28\%). Moreover, community-acquired (CA)-MSSA strains represented the leading cause of IE $(60 \%)$, followed by hospital-acquired (HA)-MRSA (25\%), while MSSA were the main pathogens 
responsible for BSI both in community-associated and in the hospital isolates (Table 1).

All strains were epidemiologically and genetically unrelated, as revealed by PFGE macro-restriction analyses (data not shown).

\section{Molecular analysis}

As reported in Table 1, the molecular analysis performed by MLST showed the spread of several hospital- and communityassociated clones, with different levels of diffusion, confirming, in part, the origin of the infections. A few exceptions were found: in isolates from the IE-CA-MRSA group, classical nosocomial isolates (ST228 SCCmec types I and IA) were identified; on the contrary, in nosocomial IE-MRSA, an increasing number of HA-MRSA strains carrying SCCmec types IV (IVa, IVc, IVh) and V, which were traditionally associated with community strains, were found (Table 2).

Generally speaking, ST5 and ST8 were uniformly represented both among MRSA and MSSA strains from both BSI and IE. Two clones often associated with community-acquired MRSA - ST30 and ST59 - were particularly diffused only among MSSA, while MRSA strains were frequently associated with the major STs already described in nosocomial MRSA strains. With regard to the SCCmec found, the SCCmec I and II were found associated predominantly with nosocomial STs, while SCCmec IV and variants (IVc, IVh) and V were associated with CA-MRSA clones. This behaviour is in agreement with the major diffusion of CA-MSSA versus HAMRSA strains, both among IE and BSI $S$. aureus strains.

Among BSI-MRSA strains, hospital-associated isolates represented the more frequent cause of infection (64\%) and were sustained by nosocomial clones, in which each SCCmec-type was always associated with a specific genetic background (suggesting clonal diffusion).

Molecular typing of CA-MRSA strains, belonging both to BSI and IE, showed genetic backgrounds more frequently associated with nosocomial clones (ST8-IV and ST228-I/IA respectively), demonstrating the potential role of nosocomial MRSA as a cause of serious infections in the community.

The $p v l$ gene was found in four strains, and it was preferentially associated with ST30 $(n=2)$ and ST5 backgrounds. A PVL-positive HA-MRSA strain belonging to ST152 carrying a SCCmec V cassette (ST152-HA-MRSA$\mathrm{V}$ clone), was identified to be responsible for enhanced severity of the disease (IE), correlating with multiple therapeutic failures and mortality.

Antibiotic resistance

Concerning the antibiotic susceptibility profiles, a low rate of resistance to non-beta-lactam drugs was observed among MSSA, while MRSA strains showed the acquisition of 
resistance to fluoroquinolones (80\%), erythromycin (42\%), gentamicin $(35 \%)$, clindamycin $(22 \%)$, rifampin $(12 \%)$ and tetracycline $(10 \%)$. There were no significant differences in antibiotic susceptibility patterns between the two groups, i.e., BSI and IE.

With regard to the susceptibilities of the major antiGram-positive drugs (Table 2), all $S$. aureus isolates showed vancomycin and teicoplanin $\mathrm{MIC}_{90}$ values of $2 \mathrm{mg} / \mathrm{l}$ in both MRSA and MSSA: the only difference between the two drugs was found in the IE-MSSA subgroup in which teicoplanin showed a $\mathrm{MIC}_{90}$ value of $1 \mathrm{mg} / \mathrm{l}$. Against all MSSA and MRSA strains, including the hVISA ones, daptomycin $\left(\mathrm{MIC}_{90} 0.5-1 \mathrm{mg} / \mathrm{l}\right)$, linezolid ( $\left.\mathrm{MIC}_{90} 2-4 \mathrm{mg} / \mathrm{l}\right)$, quinupristin/dalfopristin $\left(\mathrm{MIC}_{90}\right.$ $0.5-1 \mathrm{mg} / \mathrm{l})$, and tigecycline $\left(\mathrm{MIC}_{90} 0.25-0.5 \mathrm{mg} / \mathrm{l}\right.$ ) retained their full activity. A higher linezolid $\mathrm{MIC}_{90}$ value was found in the MSSA and MRSA strains from BSI $\left(\mathrm{MIC}_{90} 4 \mathrm{mg} / \mathrm{l}\right)$ while the $\mathrm{MIC}_{90}$ of $2 \mathrm{mg} / \mathrm{l}$ was detected in all other isolates. A reduced susceptibility to quinupristin/ dalfopristin (MIC $2 \mathrm{mg} / \mathrm{l}$ ) was observed in 5 S.aureus strains from IE (3 IE-MRSA and 2 IE-MSSA): overall, the $\mathrm{MIC}_{90}$ values of this drug was maintained in the range of susceptibility $\left(\mathrm{MIC}_{90} 0.5-1 \mathrm{mg} / \mathrm{l}\right)$.

\section{hVISA behaviour}

The screening with the Macro-Etest was applied to all strains, and the positive ones were confirmed by PAP/AUC analysis. In our laboratory, specificity and sensitivity values for the Macro-Etest method, with respect to PAP/AUC, were respectively $100 \%$ and $75 \%$ [18]. Table 2 shows their distribution among the diverse groups and the correlation with their genotypes. The hVISA phenotype was found among all S.aureus strains, greater in IE (13.1\% in MSSA and $9.2 \%$ in MRSA) than in BSI (11.5\% in MSSA and $3.8 \%$ in MRSA), but also greater in MS- than MRSA isolates. It is relevant that this heteroresistant behaviour was found in MSSA clones showing a MIC range of $0.5-1 \mathrm{mg} /$ 1 for vancomycin and $0.25-1 \mathrm{mg} / 1$ for teicoplanin. The percentage of hVISA-MSSA strains was almost double with respect to MRSA. In the MS-hVISA strains, despite lower MIC values to vancomycin and teicoplanin, few colonies were able to grow on agar plates containing 4$6 \mathrm{mg} / \mathrm{l}$ of vancomycin, which is consistent with the hVISA phenotype.

The molecular analysis of these strains revealed that among all hVISA-MSSA isolates, two major genetic backgrounds - ST8 $(n=4)$ and ST30 $(n=4)$, normally diffused in the community independently from their origin, were represented. Contrary to what was observed in MSSA strains and, as expected, the hVISA-MRSA strains belonged to the most prevalent nosocomial lineages ST228-I ( $n=5)$, ST247-IA $(n=2)$, and ST8-IA/IVc $(n=2)$. 
Clinical evaluation on therapies of hVISA infections

Overall, complete data on therapy were available for 20 out of 25 patients with an hVISA infection. The 11 patients with an MS-hVISA infection were treated with oxacillin with or without a glycopeptide, and all survived. On the contrary, 5 out of 9 patients with an MR-hVISA infection $(55 \%)$ receiving a therapy with a glycopeptide died.

\section{Discussion}

In this study we investigated the molecular characteristics and the resistance profiles of $S$. aureus isolated in persistent bacteremia and in endocarditis during 2007-2009 in four centers in Italy. Our sample comprised more MSSA than MRSA strains isolated from both infections, approximately half of them community-associated, in which CA-MSSA strains were predominant. Patients' records and settings allowed us to distinguish CA-MRSA strains from nosocomial strains, by epidemiological criteria: MLST analysis and SCCmec-typing data demonstrated the changing epidemiology of MRSA in these infections, in which the distinction between CA- and HA-MRSA is becoming increasingly blurred. This is also confirmed by the high proportions of mobile SCCmec type IV and V strains, as well as a $p v l$-positive strain among HA-MRSA [19-21]. It should be noted that the latter PVL-positive case, belonging to ST152-SCCmec V, responsible for enhanced severity of the disease (IE), correlated with multiple therapeutic failures and patient mortality. The ST152-SCCmec V clone had already been observed in Italy and in other countries, always associated with severe infections [22-24].

With only the exception of the PVL-positive HA-MRSA responsible for a severe form of IE, all other PVL-positive strains were CA-MSSA belonging to ST5 and ST30, exactly the same genetic backgrounds of the most diffuse Italian PVL+ CA-MRSA clones [25, 26].

Strains of MRSA and MSSA from both BSI and IE showed two common genetic backgrounds, ST5 and ST8; two clones, often associated with communityacquired MRSA-ST30 and ST59-were found only among MSSA, showing that community genotypes have already emerged in hospitals as a major cause both in BSI and IE, independent of their association with methicillin resistance [27].

Approximately one quarter of the isolates exhibited an hVISA phenotype. In our data, this phenomenon was not only present in MRSA strains, more frequent in IE than in BSI, in which hVISA were found in well-described nosocomial clones belonging to the agr type II [18] with vancomycin MIC of $1-2 \mathrm{mg} / \mathrm{l}$, but also in MSSA, in a lower MIC range of $0.5-1 \mathrm{mg} / \mathrm{l}$.
The study design did not include the association of the in vitro results with clinical outcomes, and the few records evaluated in the subset of hVISA infections, even if indicating a possible glycopeptides failure trend, cannot permit us to draw any definitive conclusion. The observation that $26 \%$ of S. aureus responsible for IE were hVISA at least raises some concerns about the pressure that vancomycin has exerted on these strains by prolonged subtherapeutic exposure. Furthermore, mortality data obtained from all centers in 2007-2008 reported 10\% mortality in infections caused by MSSA, including those caused by heteroresistant strains, and $28 \%$ in those by MRSA, confirming the high risk of mortality if a methicillinresistant strain was found to be the main pathogen. It is well documented that vancomycin shows a low level of eradication due to its limited penetration into the valvular vegetations [28].

With regard to reduced vancomycin susceptibility in MSSA, only a few detailed reports exist in the literature, some of them dealing with possible MRSA precursors in which mecA had been lost. Pillai et al. found hVISA in a series of clinical MSSA isolates from patients who experienced vancomycin therapy failure, and the authors concluded recommending attention in settings in which MSSA infections are treated empirically with vancomycin and in which these strains-owing to their diffuse susceptibility - are not readily detected by routine methods [29-32].

The MS-hVISA in our study belonged to different clones, many of them found in the STs of the Italian CAMRSA. In particular, the MSSA genomes of strains belonging to ST30 showed a high predisposition to be heteroresistant and PVL-positive [33]. It is very difficult to draw any conclusions on the emergence and role of these strains with reduced susceptibility to vancomycin, but one hypothesis could be the pressure exerted by widespread glycopeptide use that is able to act simultaneously on different, probably predisposed, bacterial clones.

\section{Conclusion}

In summary, this study makes several key observations. The epidemiology of MRSA is changing and the distinction between CA- and HA-MRSA is becoming increasingly blurred in Italy, as is true elsewhere in the world. Furthermore, in our strains, different MSSA isolated in BSI and IE possessed the same backgrounds as the CAMRSA found in Italy.

The successful migration of clones from the community seems to be a recent development. The SCCmec type IV strain of MRSA may present some competitive advantages over multidrug-resistant MRSA strains: it has been shown to replicate more quickly and seems to affect patients who 
are less ill [34]. On the other hand, most SCCmec type IV MRSA clones are susceptible to many non- $\beta$ lactam drugs, providing an increasing number of therapeutic options compared with the classic nosocomial strains.

The hVISA phenotype occurred more frequently in isolates from IE and in both groups of strains. As expected, hVISA was found in $a g r \mathrm{I}$ and II HA-MR clones in a vancomycin range of $1-2 \mathrm{mg} / \mathrm{l}$ [18], but our study also highlights the presence of hVISA in MSSA with vancomycin and teicoplanin susceptibility between 0.5 and $1 \mathrm{mg} / \mathrm{l}$, which demonstrated under-detecting and under-reporting because it is hidden by a profile of full susceptibility. It is necessary to emphasize that the 5 cases of glycopeptide failure in MR-hVISA infections can only confirm the need for studies evaluating the impact of hVISA in the clinical outcome of severe infections.

Acknowledgements This work was partially supported by the 'Ministero dell'Istruzione, dell'Universita' e della Ricerca' (MIUR) (project number 2008-87SM5HM).

Open Access This article is distributed under the terms of the Creative Commons Attribution Noncommercial License which permits any noncommercial use, distribution, and reproduction in any medium, provided the original author(s) and source are credited.

\section{References}

1. Hoen B, Alla F, Selton-Suty C, Béguinot I, Bouvet A, Briançon S, Casalta JP, Danchin N, Delahaye F, Etienne J, Le Moing V, Leport C, Mainardi JL, Ruimy R, Vandenesch F (2002) Changing profile of infective endocarditis: results of a 1-year survey in France. JAMA 288(1):75-81

2. Chi C, Wang SM, Lin CC, Liu CC (2010) Microbiological characteristics of community-associated Staphylococcus aureus causing uncomplicated bacteremia and infective endocarditis. J Clin Microbiol 48(1):292-294

3. Sakoulas G, Gold HS, Cohen RA, Venkataraman L, Moellering RC, Eliopoulos GM (2006) Effects of prolonged vancomycin administration on methicillin-resistant Staphylococcus aureus (MRSA) in a patient with recurrent bacteraemia. J Antimicrob Chemother 57(4):699-704

4. Sader HS, Jones RN, Rossi KL, Rybak MJ (2009) Occurrence of vancomycin-tolerant and heterogeneous vancomycin-intermediate strains (hVISA) among Staphylococcus aureus causing bloodstream infections in nine USA hospitals. J Antimicrob Chemother 64(5):1024-1028

5. Wang JL, Wang JT, Sheng WH, Chen YC, Chang SC (2010) Nosocomial methicillin-resistant Staphylococcus aureus (MRSA) bacteremia in Taiwan: mortality analyses and the impact of vancomycin, $\mathrm{MIC}=2 \mathrm{mg} / \mathrm{L}$, by the broth microdilution method. BMC Infect Dis 10:159-165

6. Bae IG, Federspiel JJ, Miró JM, Woods CW, Park L, Rybak MJ, Rude TH, Bradley S, Bukovski S, de la Maria CG, Kanj SS, Korman TM, Marco F, Murdoch DR, Plesiat P, RodriguezCreixems M, Reinbott P, Steed L, Tattevin P, Tripodi MF, Newton KL, Corey GR, Fowler VG (2009) Heterogeneous vancomycinintermediate susceptibility phenotype in bloodstream methicillinresistant Staphylococcus aureus isolates from an international cohort of patients with infective endocarditis: prevalence, genotype, and clinical significance. J Infect Dis 200(9):1355-1366

7. Entenza JM, Veloso TR, Vouillamoz J, Giddey M, Moreillon P (2011) Failure of vancomycin continuous infusion against experimental endocarditis due to vancomycin-intermediate Staphylococcus aureus. Antimicrob Agents Chemother 55(1):385-387

8. Moise PA, Smyth DS, Robinson DA, El-Fawal N, McCalla C, Sakoulas G (2009) Genotypic and phenotypic relationships among methicillin-resistant Staphylococcus aureus from three multicentre bacteraemia studies. J Antimicrob Chemother 63(5):873-876

9. Fowler VG, Miro JM, Hoen B, Cabell CH, Abrutyn E, Rubinstein E, Corey GR, Spelman D, Bradley SF, Barsic B, Pappas PA, Anstrom KJ, Wray D, Fortes CQ, Anguera I, Athan E, Jones P, van der Meer JT, Elliott TS, Levine DP, Bayer AS (2005) Staphylococcus aureus endocarditis: a consequence of medical progress. JAMA 293(24):3012-3021

10. Yoon HJ, Choi JY, Kim CO, Kim JM, Song YG (2005) A comparison of clinical features and mortality among methicillinresistant and methicillin-sensitive strains of Staphylococcus aureus endocarditis. Yonsei Med J 46(4):496-502

11. Chang FY, MacDonald BB, Peacock JE, Musher DM, Triplett P, Mylotte JM, O’Donnell A, Wagener MM, Yu VL (2003) A prospective multicenter study of Staphylococcus aureus bacteremia: incidence of endocarditis, risk factors for mortality, and clinical impact of methicillin resistance. Medicine 82(5):322-332

12. Hill EE, Peetermans WE, Vanderschueren S, Claus P, Herregods MC, Herijgers P (2008) Methicillin-resistant versus methicillinsensitive Staphylococcus aureus infective endocarditis. Eur J Clin Microbiol Infect Dis 27(6):445-450

13. Li JS, Sexton DJ, Mick N, Nettles R, Fowler VG, Ryan T, Bashore T, Corey GR (2000) Proposed modifications to the Duke criteria for the diagnosis of infective endocarditis. Clin Infect Dis 30 (4):633-638

14. Horan TC, Andrus M, Dudeck MA (2008) CDC/NHSN surveillance definition of health care-associated infection and criteria for specific types of infections in the acute care setting. Am J Infect Control 36(5):309-332

15. Clinical and Laboratory Standard Institutes (2011) Performance Standards for Antimicrobial Susceptibility Testing; Twenty-First Informational Supplement M100-S21. CLSI, Wayne, PA, USA

16. The European Committee on Antimicrobial Susceptibility Testing (EUCAST) Clinical Breakpoints (2011) http://www.eucast.org/ clinical_breakpoints (Accessed 5 May 2011)

17. Campanile F, Bongiorno D, Borbone S, Stefani S (2009) Hospitalassociated methicillin-resistant Staphylococcus aureus (HA-MRSA) in Italy. Ann Clin Microbiol Antimicrob 8:22-31

18. Campanile F, Borbone S, Perez M, Bongiorno D, Cafiso V, Bertuccio T, Purrello S, Nicolosi D, Scuderi C, Stefani S (2010) Heteroresistance to glycopeptides in Italian meticillin-resistant Staphylococcus aureus (MRSA) isolates. Int J Antimicrob Agents 36(5):415-419

19. Campanile F, Bongiorno D, Borbone S, Stefani S (2010) Methicillin-resistant Staphylococcus aureus (MRSA) evolution: the multiple facets of an old pathogen. Eur Infect Dis 4(1):7076

20. Vidal PM, Trindade PA, Garcia TO, Pacheco RL, Costa SF, Reinert C, Hiramatsu K, Mamizuka EM, Garcia CP, Levin AS (2009) Differences between "classical" risk factors for infections caused by methicillin-resistant Staphylococcus aureus (MRSA) and risk factors for nosocomial bloodstream infections caused by multiple clones of the staphylococcal cassette chromosome mec type IV MRSA strain. Infect Control Hosp Epidemiol 30(2):139-145

21. Valsesia G, Rossi M, Bertschy S, Pfyffe GE (2010) Emergence of SCCmec type IV and SCCmec type V methicillin-resistant Staphylococcus aureus containing the Panton-Valentine leukocidin genes in a large academic teaching hospital in central Switzerland: 
external invaders or persisting circulators? J Clin Microbiol 48 (3):720-727

22. Bassetti M, Nicco E, Malgorzata M, Viscoli C, Valbusa A, Bongiorno D, Campanile F, Stefani S (2010) Community associated methicillin resistant Staphylococcus aureus (CA-MRSA) infective endocarditis in Italy. J Infect 61(4):353-355

23. Okon KO, Basset P, Uba A, Lin J, Oyawoye B, Shittu AO, Blanc DS (2009) Cooccurrence of predominant Panton-Valentine leukocidin-positive sequence type (ST) 152 and multidrugresistant ST 241 Staphylococcus aureus clones in Nigerian hospitals. J Clin Microbiol 47(9):3000-3003

24. Pérez-Roth E, Alcoba-Flórez J, López-Aguilar C, GutiérrezGonzález I, Rivero-Pérez B, Méndez-Alvarez S (2010) Familial furunculosis associated with community-acquired leukocidinpositive methicillin-susceptible Staphylococcus aureus ST152. J Clin Microbiol 48(1):329-332

25. Borbone S, Campanile F, Bongiorno D, Stefani S (2010) In vitro bactericidal activity of ceftobiprole against hospital- and communityassociated methicillin-resistant Staphylococcus aureus. J Antimicrob Chemother 65(3):591-594

26. Sanchini A, Campanile F, Monaco M, Cafiso V, Rasigade JP, Laurent F, Etienne J, Stefani S, Pantosti A (2011) DNA microarray-based characterization of Panton-Valentine leukocidinpositive community-acquired methicillin-resistant Staphylococcus aureus from Italy. Eur J Clin Microbiol Infect Dis 10.1007/ s10096-011-1234-x

27. Wang CC, Lo WT, Chu ML, Siu LK (2004) Epidemiological typing of community-acquired methicillin-resistant Staphylococcus aureus isolates from children in Taiwan. Clin Infect Dis 39(4):481-487

28. Cremieux AC, Maziere B, Vallois JM, Ottaviani M, Azancot A, Raffoul H, Bouvet A, Pocidalo JJ, Carbon C (1989) Evaluation of antibiotic diffusion into cardiac vegetations by quantitative autoradiography. J Infect Dis 159(5):938-944

29. Pillai SK, Wennersten C, Venkataraman L, Eliopoulos GM, Moellering RC, Karchmer AW (2009) Development of reduced vancomycin susceptibility in methicillin-susceptible Staphylococcus aureus. Clin Infect Dis 49(8):1169-1174

30. Naimi TS, Anderson D, O'Boyle C, Boxrud DJ, Johnson SK, Tenover FC, Lynfield R (2003) Vancomycin-intermediate Staphylococcus aureus with phenotypic susceptibility to methicillin in a patient with recurrent bacteremia. Clin Infect Dis 36(12):1609 1612

31. Hageman JC, Pegues DA, Jepson C, Bell RL, Guinan M, Ward KW, Cohen MD, Hindler JA, Tenover FC, McAllister SK, Kellum ME, Fridkin SK (2001) Vancomycin-intermediate Staphylococcus aureus in a home health-care patient. Emerg Infect Dis 7(6):1023 1025

32. Bobin-Dubreux S, Reverdy ME, Nervi C, Rougier M, Bolmström A, Vandenesch F, Etienne J (2001) Clinical isolate of vancomycinheterointermediate Staphylococcus aureus susceptible to methicillin and in vitro selection of a vancomycin-resistant derivative. Antimicrob Agents Chemother 45(1):349-352

33. Rasigade JP, Laurent F, Lina G, Meugnier H, Bes M, Vandenesch F, Etienne J, Tristan A (2010) Global distribution and evolution of Panton-Valentine leukocidin-positive methicillin-susceptible Staphylococcus aureus, 1981-2007. J Infect Dis 201(10):15891597

34. Okuma K, Iwakawa K, Turnidge JD, Grubb WB, Bell JM, O'Brien FG, Coombs GW, Pearman JW, Tenover FC, Kapi M, Tiensasitorn C, Ito T, Hiramatsu K (2002) Dissemination of new methicillin-resistant Staphylococcus aureus clones in the community. J Clin Microbiol 40:4289-4294 